\title{
Planificación, programación de compras y la adquisición de equipos médicos de una institución de salud estatal, Cusco 2021
}

\author{
Raúl Villalobos Jaquehua \\ raulv14@ hotmail.com \\ Universidad César Vallejo \\ Lima-Perú
}

\section{RESUMEN}

OBJETIVO: El objetivo de la investigación fue determinar la influencia de la planificación y la programación de compras en la adquisición de equipos médicos de una institución de salud estatal, Cusco 2021. METODOLOGíA: La metodología de la investigación tuvo un enfoque cuantitativo, de tipo básica, con un diseño no experimental, las técnicas de recolección de datos la encuesta y como instrumento se realizó un cuestionario de 12 preguntas, el cual fue aplicado a 116 trabajadores de la oficina de administración, el cual tuvo una población de 164 trabajadores. RESULTADOS: Como resultado se evidenció que la planificación y la programación de compras influyen positivamente en la adquisición de equipos médicos de una institución de salud estatal, Cusco 2021, además, esta planificación y la programación de compras influyen positivamente en la adquisición de equipos médicos en situación de emergencia. CONCLUSIÓN: Finalmente, se concluye que, la implementación de la planificación y la programación de compras influyen positivamente en la adquisición de equipos médicos de una institución de salud.

Palabras clave: planificación; salud; equipos; adquisición. 


\title{
Planning, scheduling of purchases and the acquisition of medical equipment from a state health institution, Cusco 2021
}

\begin{abstract}
OBJECTIVE: The objective of the research was to determine the influence of purchasing planning and scheduling on the acquisition of medical equipment from a state health institution, Cusco 2021. METHODOLOGY: The research methodology had a quantitative approach, of a basic type, with a non-experimental design, the survey data collection techniques and a 12-question questionnaire were used as an instrument, which was applied to 116 workers from the administration, which had a population of 164 workers. RESULTS: As a result, it was evidenced that the planning and scheduling of purchases positively influence the acquisition of medical equipment from a state health institution, Cusco 2021, in addition, this planning and scheduling of purchases positively influence the acquisition of medical equipment in the situation of emergency. CONCLUSION: Finally, it is concluded that, the implementation of planning and purchasing scheduling positively influence the acquisition of medical equipment from a health institution.
\end{abstract}

Keywords: planning; health; team; acquisition.

Artículo recibido: 30 noviembre. 2021 Aceptado para publicación: 29 diciembre 2021

Correspondencia: Raulv14@ hotmail.com Conflictos de Interés: Ninguna que declarar 


\section{INTRODUCCIÓN}

Alrededor del año 2021 en una entidad de salud que pertenece al estado la cual se encuentra ubicada en Cusco, se pudo detectar como realidad problemática que cuentan con escasos equipos médicos lo cual significa que no están siendo correctamente abastecidos. El área administrativa de esta entidad no poseía una adecuada planificación o sistema que pueda para poder adquirir dichos equipos, a pesar de que en los últimos años se ha registrado una fuerte inversión en el sector salud; sin embargo, se verifica que aún se requieren de más fondos, consecuencia de la coyuntura actual por la que estamos atravesando, puesto que ahora se necesitan cubrir más necesidades que presentan los ciudadanos, y contar con suficiente equipo médico puede ser un factor determinante para salvar una vida (Ministerio de Salud, 2021).

Oluyase et al. (2021) mencionan que desde inicios del año 2020, se viene viviendo una situación bastante complicada por la llegada de la COVID-19, lo que llevo a dejar en evidencia las malas gestiones que se realizaban en las instituciones de salud, puesto que ya contaban con varias deficiencias y tras la llegada de este virus, esta problemática se acentuó más, siendo así que se considere indispensable que el Estado preste más atención a este sector.

Bousquat y Baffini de Paula (2017) mencionan que es importante que los centros de salud sean óptimos para todos los pacientes ya que deben recibir la atención necesaria y en buenas condiciones, la propuesta de ejecución de las regiones de salud y redes está ganando relevancia política, con el objetivo de luchar contra la fragmentación de la atención, aumentar el acceso y asegurar la equidad y la universalidad.

Asimismo, por falta de una adecuada planificación por parte de la administración de las instituciones de salud, no se podido elaborar una estrategia que desarrolle una adecuada adquisición de maquinaria médica, lo que ha conllevado a que los pacientes se vean perjudicados en su atención; lo que resulta en, agravamientos de su salud, demoran en dicha atención, así como su mala calidad (Mohammadfam et al.,2017).

Ademàs, es importante mencionar que la planificación viene a ser el proceso en el cual se definen las actividades que buscaran alcanzar objetivos diseñados en relación a la misión y visión de las organizaciones. De igual manera íntegra aspectos en los que se evalúan situaciones futuras y las posibles actuaciones frente a ellas, y así establecer procedimientos en áreas donde se identifiquen debilidades para posteriormente aplicar 
cambios que las instituciones necesitan para mejorar su calidad en la gestión y como consecuencia también sus servicios (Papke y Boyer,2017). En la misma idea, Sánchez (2017) precisó que la planificación se caracteriza por plantear la formulación del alcance de los objetivos en un lapso de mediano a largo plazo, contando también, con las pequeñas metas que se van alcanzando en el camino, y formulando diferentes estrategias para el éxito de estos; de igual forma, tener conocimiento sobre de las políticas públicas y las normativas del sector, y la metodología con la que se trabaja.

Del mismo modo, es importante mencionar que la programación de compras tiene como fin adquirir aprovisionamiento, acerca de las necesidades de la compra, de tal manera, también el mantenimiento del nivel de servicio. De tal manera que una buena gestión de compras puede suponer el incremento de beneficios, es decir consiste en buscar herramientas de suministro para poder adquirir las mercancias necesarias que influyan en el desarrollo de la actividad empresarial, y consecuentemene satisfacer la demanda (Mohammadi et al.,2020). Por otra parte, Barber et al. (2018) refieren que viene a ser el proceso en el cual se definen las necesidades, los recursos disponibles y la realidad organizacional para poder definir un procedimiento de adquisición de elementos en coordinación con las unidades de abastecimiento y poder cubrir la demanda de equipos o materiales. Además, toda programación que tenga que ver con compras públicas, se basa siempre en la cantidad de presupuesto y se sigue una ideología, aunque no siempre suele seguirse. Sin embargo, debido a los escasos recursos y en busca de gastar lo que se tiene con el fin de abastecerse lo cual ocasiona que no se llegue a comprar lo que se necesita. Por otra parte, la adquisición de equipos médicos es considerada como una etapa dentro de los módulos de procesos financieros y presupuestales. En dicha etapa se pueden encontrar los planes y la programación de compras dividiéndose en necesidades, prioridades y la disponibilidad que haya en los recursos, de esta forma poder otorgar calidad y seguridad de los pacientes, así como hacer más eficientes los procedimientos internos y ofrecer una mayor cobertura al mejorar la capacidad de respuesta en emergencias (Suwantarat et al.,2017). En este contexto los diversos equipos médicos se han vuelto herramientas muy importantes para poder ayudar a los especialistas de la salud a poder hacer un diagnóstico más preciso y poder tratar de manera correcta y eficaz las múltiples enfermedades que hoy en día existen, dándole a los pacientes un servicio de calidad (Camacho et al., 2017). 
Del mismo modo, Bahreini et al. (2019) señalan que toda adquisición de cualquier bien debe aplicar distintos mecanismos e ir actualizándolos para que se obtenga una mejora en el direccionamiento de las necesidades que hay por cubrir, la gestión eficaz del mantenimiento de estos y las reparaciones a las que deben someterse cada cierto tiempo deberán planificarse y aplicarse mediante estrategias de mantenimiento adecuadas de esta manera asegurar que estos dispositivos estarán seguros y seguirán funcionando correctamente.

Es así que, según lo precisado anteriormente, se infiere que la institución de salud estatal requiere de una mejora en su planificación, organización y en su programación de compras para poder adquirir equipos médicos con el fin satisfacer las expectativas de los pacientes y poder asistirlos en todas las necesidades que presenten.

\section{Problema General}

- ¿Cómo influye la planificación y la programación de compras en la adquisición de equipos médicos de una institución de salud estatal, Cusco 2021?

\section{Problemas específicos}

- ¿Cómo influye la planificación y la programación de compras en la evaluación de tecnologías para la adquisición de equipos médicos de una institución de salud estatal, Cusco, 2021 ?

- ¿Cómo influye la planificación y la programación de compras en la evaluación de dispositivos para la adquisición de equipos médicos de una institución de salud estatal, Cusco, 2021?,

- ¿Cómo influye la planificación y la programación de compras en la instalación de equipos médicos de una institución de salud estatal, Cusco, 2021?

\section{Objetivo General}

- Determinar la influencia de la planificación y la programación de compras en la adquisición de equipos médicos de una institución de salud estatal, Cusco 2021.

\section{Objetivos específicos}

- Determinar la influencia de la planificación y la programación de compras en la evaluación de tecnologías para la adquisición de equipos médicos de una institución de salud estatal, Cusco, 2021. 
- Determinar la influencia de la planificación y la programación de compras en la evaluación de dispositivos para la adquisición de equipos médicos de una institución de salud estatal, Cusco, 2021.

- Determinar la influencia de la planificación y la programación de compras en la instalación de equipos médicos de una institución de salud estatal, Cusco, 2021.

\section{Hipótesis General}

- La planificación y la programación de compras influyen positivamente en la adquisición de equipos médicos de una institución de salud estatal, Cusco 2021.

\section{Hipótesis específicas}

- La planificación y la programación de compras influyen positivamente en la evaluación de tecnologías para la adquisición de equipos médicos de una institución de salud estatal, Cusco, 2021.

- La planificación y la programación de compras influyen positivamente en la evaluación de dispositivos para la adquisición de equipos médicos de una institución de salud estatal, Cusco, 2021.

- La planificación y la programación de compras influyen positivamente en la instalación de equipos médicos de una institución de salud estatal, Cusco, 2021.

\section{Antecedentes}

El autor Smith (2019), en su trabajo de investigación "Healthcare Organizations and Succession Planning", tuvo como objetivo, investigar como las instituciones de salud elaboran sus planificaciones y si es que se hace de manera correcta. Se contó con un estudio de enfoque cualitativo; y, se concluyó que, dentro de los sistemas de salud, hay una alta necesidad por implementar planes que sean exitosos ya que, sin estos se encontrarán enredados en demasiados problemas sin solución inmediata y que, dichos planes no están siendo ejecutados en la cantidad que se debería, cosa que traería incrementos en los costos de los hospitales y entidades de salud.

Del mismo modo, Erondu et al. (2018) tuvo como objetivo en su investigaciòn informar sobre la necesidad de sistemas de salud sólidos; sin embargo, el acuerdo es menos evidente sobre los mecanismos efectivos para establecer y mantener estos sistemas, particularmente en entornos con recursos limitados y en presencia de tensiones múltiples y sostenidas, por ejemplo, conflictos, hambrunas, cambio climático y globalización. La elaboración de las directrices del Reglamento Sanitario Internacional (2005) y el marco 
de los sistemas de salud de la OMS ha dado lugar a la separación de las funciones de salud pública y los servicios, que en realidad son interdependientes y deben integrarse para garantizar una salud nacional continua e ininterrumpida. Al analizar los esfuerzos para fortalecer los sistemas de salud y las inversiones en seguridad sanitaria mundial, se examinó las áreas de superposición y ofrecemos recomendaciones para la construcción de un sistema nacional de salud unificado que incluya la salud pública.

Por otro lado, McGreevy et al. (2019) tuvo como objetivo investigar la competitividad económica global cuando se incluye junto con los objetivos sociales inspirados en la imagen, como la habitabilidad y los objetivos ambientales, como la sostenibilidad, puede conducir a políticas que tengan un impacto positivo en la salud. También mencionó la importancia de la planeación estratégica en los establecimientos de salud lo cual favorece a un mejor gobierno y a la misma ciudadanía para recibir una atención adecuada y en óptimas condiciones. Y se obtuvo como conclusión que la planificación estratégica brinda oportunidades para el avance de la salud en el desarrollo urbano. Sin embargo, cuando está impulsado por los objetivos de productividad o habitabilidad como mejora de la imagen, puede potencialmente exacerbar las inequidades en salud.

Asimismo, Santos (2017) tuvo como objetivo evidenciar que este tipo de organización es fundamental, dado que Brasil está organizado como Federación y los tres niveles de gobierno son, en un sentido amplio, igualmente responsables de la salud. Los servicios y acciones de salud son un conjunto complejo de actividades que se interconectan en nombre de la salud ciudadana, que es un concepto global que no se puede dividir. Por lo tanto, los servicios de salud deben organizarse sistemáticamente para servir a todos por igual, independientemente del lugar donde viva el ciudadano. Esta organización sistémica requiere una interacción permanente entre las unidades federativas para discutir y operacionalizar los servicios de referencia, el financiamiento y otros aspectos técnicos y administrativos.

Además, Diaconu (2016) preciso que los dispositivos y equipos médicos de los países de ingresos bajos se encuentran de condiciones deplorables, por lo cual se necesitan más orientaciones sobre cómo llevar a cabo el proceso de adquisición de equipos médicos que cumplan con su función como es debido. A veces por la falta de recursos se opta por comprar los equipos más baratos pero que lamentablemente no son adecuados o al poco tiempo dejan de funcionar. Es necesario cumplir con las normas metodológicas impuestas 
a nivel internacional para un correcto funcionamiento de las entidades médicas en las cuales su prioridad siempre deba ser brindarles una buena atención a sus pacientes.

Finalmente, Abdul et al. (2018) tuvo como objetivo proporcionar una selección óptima de ubicaciones para las oficinas de la HHC, los trabajadores de la salud y centros de grupos de pacientes, además, de su asignación específica. refieren que la disponibilidad de una atención médica centrada en el paciente, rentable y orientada a la calidad es una tarea enorme para los planificadores de la atención médica en todos los países. En este estudio, se introduce un problema de planificación de atención médica para integrar los problemas de dimensionamiento de recursos y los aspectos de asignación con la atención basada en telesalud y los servicios de atención de los pacientes. Posteriormente, con el fin de validar la efectividad del enfoque de integración propuesto, empleamos los medios c difusos para describir la organización adecuada de las oficinas de la HHC, los trabajadores de la salud y los datos del paciente. Finalmente, se realiza un análisis de sensibilidad para explorar el comportamiento del modelo frente a la variación en los valores de los parámetros.

\section{ESTRATEGIAS METODOLÓGICAS O MATERIALES Y MÉTODOS}

La presente investigación se realizó con un enfoque cuantitativo,de acuerdo con Guedes dos Santos et al. (2017), señalo que una investigación cuantitativa se basa en la elaboración de estudios que están vinculados a datos númericos, los cuales fomentan la conjunción de detalles e información de esta manera permita corroborar teorias y/o hipótesis.En la misma linea Tobi y Kampen (2018) señalan que esta permitió evidenciar los resultados de manera numérica y cuantificar los productos en aspectos estadísticos, asimismo recopilar información a manera de verificar o corroborar las hipótesis y/o teorías planteadas.

\section{Tipo de Investigación}

El tipo de investigación utilizado fue de carácter básico y se caracterizó por profundizar con más información en el tema principal, asimismo exponer un marco teórico representativo (Lai, 2018).

\section{Diseño de investigación}

El diseño empleado en la presente investigación fue no experimental de nivel descriptivo explicativo el cual permitió emplear un metodo de analisis escensial para la representación de los resultados, asimismo evaluar los componentes que engloba el 
estudio desde la perspectiva metodologica, como la población y/o muestra (Queirós et al., 2017).

\section{Población y Muestra}

- Como población se consideró a 164 trabajadores de la oficina de administración. Esta viene a ser la cantidad de individuos que se considera para el estudio debido a que cumplen con los requisitos establecidos para llevar a cabo la investigación.

- Como muestra se seleccionó 116 trabajadores de la oficina de administración. Esta viene a ser una representación de la población previamente escogida, con el fin de recolectar la información necesaria y lo más precisa posible.

\section{Técnica e Instrumento de Recolección de Datos}

La técnica utilizada para llevar a cabo la investigación, es la encuesta en escala de Likert y como instrumento de recolección de datos el cuestionario, uno para cada variable, el cual será aplicado a la muestra en estudio .

\section{Procedimientos}

La recolección de información se realiza en tres pasos, el primer paso se centra en obtener datos importantes acerca del centro en investigación y de los sujetos de estudio, asimismo, se obtuvo un permiso de datos e información general, de modo que estos datos sean confidenciales. El segundo paso se centra en el contraste de fuentes en base a información recolectada de otras investigaciones, nacionales e internacionales, esto para que el presente estudio pueda tener información variada y verídica. El tercer paso se centra en la realización de un cuestionario acerca de las variables, de tal manera que la muestra en estudio pueda resolver las dudas y brindar información de primera mano, es importante señalar que fue validado debidamente por los expertos en base a la prueba Binominal, seguidamente se analizará en una base de datos, mediante uso de tablas y gráficos.

\section{Método de Análisis de Datos}

Asimismo, se utilizó la escala de Likert para que la información sea ordenada y precisa, y como instrumento de recolección el cuestionario. Del mismo modo, para las respuestas de los instrumentos se usó como tabulación el programa estadístico SPSS V26, con la ayuda del método estadístico descriptivo: El cual es un método que permite que la investigación pueda obtener tablas y gráficos con los datos recogidos. Por último, se hizo uso del método estadístico inferencial, esto con el fin de contrastar las hipótesis de investigación planteadas en base al Alfa de Cronbach. 


\section{RESULTADOS Y DISCUSIÓN}

\section{Resultados}

A continuación, se describen los resultados mediante tablas que contienen datos recopilados a partir de las encuestas aplicadas y realizadas a la muestra de investigación, estas se encuentran procesadas de acuerdo a las variables.

\section{HIPÓTESIS GENERAL}

H1: La planificación y la programación de compras influyen positivamente en la adquisición de equipos médicos de una institución de salud estatal, Cusco 2021.

Ho: La planificación y la programación de compras NO influyen positivamente en la adquisición de equipos médicos de una institución de salud estatal, Cusco 2021.

Tabla 1: Información de ajuste de los modelos de la hipótesis general.

\begin{tabular}{|l|c|c|c|c|c|c|}
\hline \multirow{2}{*}{ Modelo } & \multicolumn{4}{|c|}{ Criterios de ajuste de modelo } & \multicolumn{3}{c|}{$\begin{array}{c}\text { Pruebas de la razón de } \\
\text { verosimilitud }\end{array}$} \\
\cline { 2 - 7 } & AIC & BIC & $\begin{array}{l}\text { Logaritmo de la } \\
\text { verosimilitud - }\end{array}$ & $\begin{array}{c}\text { Chi- } \\
\text { cuadrado }\end{array}$ & gl & Sig. \\
\hline Sólo intersección & 588,609 & 635,420 & 554,609 & & & \\
Final & 518,960 & 659,393 & 416,960 & 137,649 & 34 &, 000 \\
\hline
\end{tabular}

Nota: Elaboración propia.

Interpretación: Se aprecia en la tabla 1 que Con X2 = 137,649, gl = 34 y p = 0.000; el valor de $\mathrm{p}$ (sig) fue menor que $0,05(0,000<0,05)$ entonces se rechaza la hipótesis nula H0 y se acepta la hipótesis planteada por el investigador H1: "La planificación y la programación de compras influyen positivamente en la adquisición de equipos médicos de una institución de salud estatal, Cusco 2021"; asimismo, al tener el valor de 0,699 en el Pseudo R-cuadrado de Nagelkerke, el mismo que se encuentra cercano al 1, afirmamos que existe una influencia moderada de las variables planificación y programación de compras referente a la adquisición de equipos médicos dentro de una institución estatal.

\section{Hipótesis Específicas}

\section{Hipótesis Específica 1}

H1: La planificación y la programación de compras influyen positivamente en la evaluación de tecnologías para la adquisición de equipos médicos de una institución de salud estatal, Cusco, 2021.

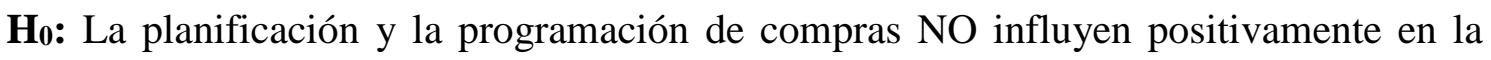
evaluación de tecnologías para la adquisición de equipos médicos de una institución de salud estatal, Cusco, 2021. 
Tabla 2: Información de ajuste de los modelos de la hipótesis específica 1.

\begin{tabular}{|l|c|c|c|c|c|c|}
\hline \multirow{2}{*}{ Modelo } & \multicolumn{4}{|c|}{ Criterios de ajuste de modelo } & \multicolumn{3}{c|}{$\begin{array}{c}\text { Pruebas de la razón de } \\
\text { verosimilitud }\end{array}$} \\
\cline { 2 - 7 } & AIC & BIC & $\begin{array}{l}\text { Logaritmo de la } \\
\text { verosimilitud -2 }\end{array}$ & $\begin{array}{c}\text { Chi- } \\
\text { cuadrado }\end{array}$ & gl & Sig. \\
\hline Sólo intersección & 394,008 & 413,283 & 380,008 & & & \\
Final & 299,677 & 357,502 & 257,677 & 122,331 & 14 &, 000 \\
\hline
\end{tabular}

Nota: Elaboración propia.

Interpretación: Se aprecia en la tabla 2 con X2 = 122,331, gl = 14 y p = 0.000; el valor de $\mathrm{p}$ (sig) fue menor que $0,05(0,000<0,05)$ entonces se rechaza la hipótesis nula H0 y se acepta la hipótesis planteada por el investigador H1: "La planificación y la programación de compras influyen positivamente en la evaluación de tecnologías para la adquisición de equipos médicos de una institución de salud estatal, Cusco, 2021"; asimismo, al tener el valor de 0,672 en el Pseudo R-cuadrado de Nagelkerke, el mismo que se encuentra cercano al 1 , afirmamos que existe una influencia moderada de las variables planificación y programación de compras referente a la evaluación de tecnologías para la adquisición de equipos médicos de una institución de salud estatal

\section{Hipótesis Específica 2}

H1: La planificación y la programación de compras influyen positivamente en la evaluación de dispositivos para la adquisición de equipos médicos de una institución de salud estatal, Cusco, 2021.

Ho: La planificación y la programación de compras NO influyen positivamente en la evaluación de dispositivos para la adquisición de equipos médicos de una institución de salud estatal, Cusco, 2021.

Tabla 3: Información de ajuste de los modelos de la hipótesis específica 2.

\begin{tabular}{|l|c|c|c|c|c|c|}
\hline \multirow{2}{*}{ Modelo } & \multicolumn{3}{|c|}{ Criterios de ajuste de modelo } & \multicolumn{3}{c|}{$\begin{array}{c}\text { Pruebas de la razón de } \\
\text { verosimilitud }\end{array}$} \\
\cline { 2 - 7 } & AIC & BIC & $\begin{array}{c}\text { Logaritmo de la } \\
\text { verosimilitud -2 }\end{array}$ & $\begin{array}{c}\text { Chi- } \\
\text { cuadrado }\end{array}$ & gl & Sig. \\
\hline Sólo intersección & 444,765 & 466,794 & 428,765 & & & \\
Final & 315,777 & 381,863 & 267,777 & 160,988 & 16 &, 000 \\
\hline
\end{tabular}

Nota: Elaboración propia.

Interpretación: Se aprecia en la tabla 3 con X2 = 160,988, gl = 16 y p = 0.000; el valor de $\mathrm{p}$ (sig) fue menor que $0,05(0,000<0,05)$ entonces se rechaza la hipótesis nula H0 y 
se acepta la hipótesis planteada por el investigador $\mathrm{H} 1$ : "La planificación y la programación de compras influyen positivamente en la evaluación de dispositivos para la adquisición de equipos médicos de una institución de salud estatal, Cusco, 2021"; asimismo, al tener el valor de 0,765 en el Pseudo R-cuadrado de Nagelkerke, el mismo que se encuentra cercano al 1, afirmamos que existe una influencia alta de las variables planificación y programación de compras referente a la evaluación de dispositivos para la adquisición de equipos médicos de una institución de salud estatal.

\section{Hipótesis específica 3}

H1: La planificación y la programación de compras influyen positivamente en la instalación de equipos médicos de una institución de salud estatal, Cusco, 2021.

Ho: La planificación y la programación de compras NO influyen positivamente en la instalación de equipos médicos de una institución de salud estatal, Cusco, 2021.

Tabla 2: Información de ajuste de los modelos de la hipótesis específica 3.

\begin{tabular}{|l|c|c|c|c|c|c|}
\hline \multirow{2}{*}{ Modelo } & \multicolumn{3}{|c|}{ Criterios de ajuste de modelo } & \multicolumn{3}{c|}{$\begin{array}{c}\text { Pruebas de la razón de } \\
\text { verosimilitud }\end{array}$} \\
\cline { 2 - 7 } & AIC & BIC & $\begin{array}{l}\text { Logaritmo de la } \\
\text { verosimilitud -2 }\end{array}$ & $\begin{array}{c}\text { Chi- } \\
\text { cuadrado }\end{array}$ & gl & Sig. \\
\hline $\begin{array}{l}\text { Sólo intersección } \\
\text { Final }\end{array}$ & 356,330 & 372,852 & 344,330 & & & \\
318,532 & 368,097 & 282,532 & 61,798 & 12 &, 000 \\
\hline
\end{tabular}

Nota: Elaboración propia.

Interpretación: Se aprecia en la tabla 3 con X2 =61,798, gl = 12 y p = 0.000; el valor de $\mathrm{p}$ (sig) fue menor que $0,05(0,000<0,05)$ entonces se rechaza la hipótesis nula H0 y se acepta la hipótesis planteada por el investigador H1: "La planificación y la programación de compras influyen positivamente en la instalación de equipos médicos de una institución de salud estatal, Cusco, 2021"; asimismo, al tener el valor de 0,431 en el Pseudo R-cuadrado de Nagelkerke, el mismo que se encuentra cercano al 1, afirmamos que existe una influencia media de las variables planificación y programación de compras referente a la instalación de equipos médicos de una institución de salud estatal, Cusco, 2021.

\section{DISCUSIÓN}

La autora Diaconu (2016) investiga el déficit que presentan los países de ingresos bajos debido a la falta equipos tecnológicos y médicos, ya que se opta por la compra de aquellos de baja calidad que, en lugar de aportar, ocasionan retrasos y brindan una 
mala atención a los clientes. EL autor precisó como resultado que se debe implementar de manera urgente el sector salud de buenos suministros y bienes médicos, ya que es uno de los más importantes en cuanto satisface la necesidad médica asistencial del país, asimismo, basándose en la revisión de la literatura, la cual establece que se debe tener un plan de contingencia y rigurosidad para seleccionar y evolucionar nuevas estrategias. Otro resultado obtenido es que en Gambia y Rumania la adquisición de equipos se encuentra impulsada por el sector privado, quienes, si predisponen de los recursos necesarios para la adquisición respectiva, además se precisó que hay una gran diferencia frente al sector público en cuanto a recursos y adquisiciones. Los resultados de Diaconu llegan a relacionarse a los de esta investigación, ya que se evidencia que una correcta planificación y programación de compras influyen positivamente en la adquisición de equipos, lo cual se puede ver en nuestra investigación ya que el 56,0\% manifestaron estar totalmente de acuerdo que es fundamental que en la cartera de pedidos, sea atendida de acuerdo al número de llegada y que debe ser atendida de manera inmediata, mientras que el 27,6\% están ni de acuerdo ni en desacuerdo.

Por otra parte, Smith (2019) en su investigación 'Healthcare organizations and succession planning" precisa que es necesario contar con planes de sucesión respecto al liderazgo de los trabajadores del centro de salud, esto con la finalidad de que una persona dirija la organización sanitaria del lugar a fin de brindar una atención idónea a los pacientes y causar satisfacción entre los empleados. El autor precisa que los sistemas de transferencia de liderazgo permiten que las organizaciones se desarrollen de manera interna tanto en la planificación, adquisición y otros temas relacionados al centro de salud, así como permite el crecimiento profesional del personal, el desarrollo de estrategias y la formación de nuevos líderes, ello con la finalidad de seguir brindando una atención médica de calidad. Lo obtenido por el autor se relaciona a los presentes resultados, ya que el 63,8\% manifestaron estar de acuerdo que se debe observar de manera continua el sistema de seguimiento para así evitar alguna demora en la recepción, por lo que solo el 10,3\% mencionaron estar totalmente en desacuerdo con lo dicho anteriormente. 


\section{CONCLUSIÓN O CONSIDERACIONES FINALES}

- Se determinó que la planificación y la programación de compras influyen positivamente en la adquisición de equipos médicos de una institución de salud estatal, Cusco 2021. Mediante el pseudo R cuadrado de Nagelkerke, se aceptó la hipótesis general planteada por el investigador ya que se obtuvo el dato de 0,699, el cual permite interpretar que existe influencia moderada entre las variables.

- Se determinó que la planificación y la programación de compras influyen positivamente en la evaluación de tecnologías para la adquisición de equipos médicos de una institución de salud estatal, Cusco, 2021. Mediante el pseudo R cuadrado de Nagelkerke se aceptó la hipótesis planteada por el investigador, ya que se obtuvo el resultado de 0,672, el cual demuestra que existe influencia moderada entre las variables; además el nivel de significancia es menor a 0,05 por lo que se acepta la hipótesis planteada.

- Se determinó que la planificación y la programación de compras influyen positivamente en la evaluación de dispositivos para la adquisición de equipos médicos de una institución de salud estatal, Cusco, 2021. Mediante el pseudo R cuadrado de Nagelkerke se aceptó la hipótesis planteada por el investigador ya que se obtuvo el dato de 0,765 , el cual señala que existe influencia alta entre las variables; así mismo el nivel de significación obtenido 0,000 permite aceptar la hipótesis planteada.

- Se determinó que la planificación y la programación de compras influyen positivamente en la instalación de equipos médicos de una institución de salud estatal, Cusco, 2021. Mediante el pseudo R cuadrado de Nagelkerke se aceptó la hipótesis planteada por el investigador, ya que se obtuvo el resultado de 0,431 , el cual señala que existe influencia media entre las variables.

\section{LISTA DE REFERENCIAS}

Abdul Nasir, J., Hussain, S., \& Chuanging, D. (2018). An Integrated Planning Approach Towards Home Health Care, Telehealth and Patients Group Based Care. Journal of Network and Computer Applications, 117, 30-41. doi:https://doi.org/10.1016/j.jnca.2018.05.009

Bahreini, R., Doshmangir, L., \& Imani, A. (2019). Bahreini, R., Doshmangir, L., \& Imani, A. (2019). Influential factors on medical equipment maintenance 
management: In search of a framework. Journal of Quality in Maintenance Engineering, 25(1), 128-143. doi:https://doi.org/10.1108/JQME-11-2017-0082

Barber, S., Kumar, A., Roubal, T., Colombo , F., \& Lorenzonid, L. (2018). Harnessing the private health sector by using prices as a policy instrument: Lessons learned from South Health 122. doi:https://doi.org/10.1016/j.healthpol.2018.03.018

Bousquat, A., \& Baffini de Paula, D. (2017). Primary health care and the coordination of care in health regions: managers' and users' perspective. Collective Science \& Saude, 27(4). doi:https://doi.org/10.1590/1413-81232017224.28632016

Camacho, J., Torres , D., \& Chavarria, T. (2019). Gestión de equipos médicos: implementación y validación de una herramienta de auditoría. Revista mexicana de ingeniería biomédica, $\quad 38(1), \quad$ 76-92. http://www.scielo.org.mx/pdf/rmib/v38n1/2395-9126-rmib-38-01-76.pdf

Diaconu, K. (2016). Methods for medical device and equipment procurement in low and middle-income countries. [Tesis de doctorado, The University of Birmingham]. https://etheses.bham.ac.uk/id/eprint/7063/1/Diaconu16PhD_redacted.pdf

Erondu, N., Martin, J., Marten, R., \& Yates, R. (2018). Building the case for embedding global health security into universal health coverage: a proposal for a unified health system that includes public health. The Lancet, 392, 1482-1486. doi:https://doi.org/10.1016/S0140-6736(18)32332-8

Guedes dos Santos, J., Lorenzini Erdmann, A., Schilindwein Meirelles, B., Marcelino de Melo , G., Pecini da Cunha, V., \& Ratchneewan, R. (2017). Integrating Quantitative and Qualitative data in mixed methods research. Texto \& Contexto Enfermagem, 26(3). doi:https://doi.org/10.1590/0104-07072017001590016

Lai, P. (2018). Research methodology for novelty technology. Journal of Information Systems and Technology Management, 15, 1-17. doi:https://doi.org/10.4301/S1807-1775201815010

McGreevy, M., Harris, P., Delany, T., Fisher, M., Sainsbury, P., \& Fran, B. (2019). Can health and health equity be advanced by urban planning strategies designed to advance global competitiveness? Lessons from two Australian case studies. Social Science \& Medicine, 242. doi:https://doi.org/10.1016/j.socscimed.2019.112594 
Ministerio de Salud. (2021). Tiempos de pandemia 2020-2021. http://bvs.minsa.gob.pe/local/MINSA/5485.pdf

Mohammadfam, I., Kamalinia, M., Momeni, M., Golmohammadi, R., Hamidi, Y., \& Soltanian, A. (2017). Evaluation of the Quality of Occupational Health and Safety Management Systems Based on Key Performance Indicators in Certified Organizations. Safety and Health at Work, 8(2), 156-161. doi:https://doi.org/10.1016/j.shaw.2016.09.001

Mohammadi, M., Emaelian, M., \& Atighehchian, A. (2020). Design of mathematical models for the integration of purchase and production lot-sizing and scheduling problems under demand uncertainty. Applied Mathematical Modelling, 84, 1-18. doi:https://doi.org/10.1016/j.apm.2020.03.021

Oluyase, A., Hocaoglu, M., Maddocks, M., \& Walshe, P. (2021). The Challenges of Caring for People Dying From COVID-19: A Multinational, Observational Study (CovPall). Journal of Pain and Symptom Management, 62(3), 460-470. doi:https://doi.org/10.1016/j.jpainsymman.2021.01.138

Papke-Shields , K., \& Boyer-Wright, K. (2017). Strategic planning characteristics applied to project management. International Journal of Project Management, 35(2), 169-179. doi:https://doi.org/10.1016/j.ijproman.2016.10.015

Queiròs , A., Faria, D., \& Almeid, F. (2017). Strengths and limitations of qualitative and quantitative research methods. European Journal of education studies, 3(9), 369387. https://oapub.org/edu/index.php/ejes/article/view/1017

Sànchez Jacas , I. (2017). La planeación estratégica en el sistema de salud cubano. MEDISAN ， 21(5), 1-7. http://scielo.sld.cu/scielo.php?script=sci_arttext\&pid=S102930192017000500019

Santos, L. (2017). Healthcare regions and their care networks: an organizationalsystemic model for SUS. Collective Science \& Saude, 27(4). doi:https://doi.org/10.1590/1413-81232017224.26392016

Smith , M. (2019). Healthcare organizations and succession planning. University of New England. Obtenido de https://dune.une.edu/theses/247

Suwantarat, N., Supple, L., Cadnum, J., \& Sankar, T. (2017). Quantitative assessment of interactions between hospitalized patients and portable medical equipment and 
other fomites. American Journal of Infection Control, 45(11), 1276-1278. doi:https://doi.org/10.1016/j.ajic.2017.05.003

"Tobi , H., \& Kampen, J. (2018). Research design: the methodology for interdisciplinary research framework. Quality \& Quantity, 52, 1209-1255. doi:https://doi.org/10.1007/s11135-017-0513-8" 